\title{
An Implicit Decoupling for the Dilatons and the Axions of the Heterotic String
}

\author{
Nejat T. Yılmaz \\ Department of Mathematics and Computer Science, \\ Çankaya University, \\ Öğretmenler Cad. No:14, 06530, \\ Balgat, Ankara, Turkey. \\ ntyilmaz@cankaya.edu.tr
}

July 23, 2018

\begin{abstract}
A set of consistency conditions is derived for the massless sector of the $D$-dimensional $E_{8} \times E_{8}$ heterotic string. Under the solvable Lie algebra gauge these conditions are further formulated explicitly in terms of the dilatons and the axions. It is then shown that these consistency conditions which are satisfied by the solution space give way to an implicit decoupling between the coset scalar sector namely the dilatons and the axions and the gauge fields of the theory.
\end{abstract}

\section{Introduction}

The global (rigid) symmetry of the scalar sectors of the supergravity theories can be extended to be the global symmetry of the entire bosonic sector of 
the theory. The global symmetries of the supergravities give us the nonperturbative U-duality symmetries of the relative string theories [1, 2, 3]. Therefore understanding the role of the scalars in the supergravity dynamics has implications not only on its own right but it will also help us to understand the duality nature of the string theories.

The ten dimensional $\mathcal{N}=1$ type I supergravity theory which is coupled to the Yang-Mills theory [4, 5] is the low energy effective limit or the massless sector of the type I superstring theory and the heterotic string theory [1, 6]. When the coupling Yang-Mills multiplet number is $N=16$, the $D=10$ Yang-Mills supergravity has the $E_{8} \times E_{8}$ gauge symmetry thus it corresponds to the low energy effective limit of the $E_{8} \times E_{8}$ ten dimensional heterotic string theory which forms the massless background coupling [1]. Due to the general Higgs vacuum structure the full symmetry $E_{8} \times E_{8}$ can be broken down to its maximal torus subgroup $U(1)^{16}$. In this case we have the low energy effective theory of the fully Higgsed ten dimensional $E_{8} \times E_{8}$ heterotic string. In [6] the Kaluza-Klein compactification of the bosonic sector of the ten dimensional $\mathcal{N}=1$ simple supergravity that is coupled to $N$ abelian gauge multiplets on the tori $T^{10-D}$ is performed and the $D$-dimensional fully Higgsed effective bosonic heterotic string lagrangian is obtained. It is also shown in the same work that when a single scalar is decoupled from the others, the rest of the scalars of the $D$-dimensional theory can be formulated as $G / K$ symmetric space sigma model. Since the global symmetry groups of the compactified $D$-dimensional effective heterotic string are non-compact real forms one can make use of the solvable Lie algebra gauge [7] to parametrize the scalar cosets and to construct the scalar lagrangians. In such a gauge simply the gauge forming solvable Lie algebra is composed of certain Cartan and positive root generators of the Lie algebra of $G$ [8].

In this work starting from the $D$-dimensional effective bosonic heterotic string lagrangian derived in [6] we show that the field equation of the single decoupled dilaton generates a set of consistency conditions which are satisfied 
by the elements of the solution space of the theory. We interpret these hidden consistency conditions as an implicit decoupling between the $G / K$ coset scalars and the $U(1)$ gauge fields. Therefore we also prove that the coset scalar solution space of the $D$-dimensional effective heterotic string is embedded in the solution space of the pure $G / K$ non-linear sigma model.

\section{Consistency Conditions and the Scalar Mat- ter Decoupling}

In this section starting from the bosonic lagrangian of the $D$-dimensional fully Higgsed low energy effective heterotic string [6] we will show that the field equation of the decoupled dilaton leads to two sets of consistency conditions which are satisfied by the solution space of the theory. Although these conditions are in general valid for generic coset scalar fields which are defined without specifying a gauge for the coset parametrization we will choose the solvable Lie algebra parametrization [7] to further simplify these conditions. By assuming a matrix representation for the Lie algebra of the global symmetry group we will express the general consistency conditions in terms of the dilatons and the axions of the theory. Then we will show that these consistency conditions reveal a hidden decoupling of the gauge fields in the field equations of the coset scalars although there exists a scalar-matter coupling term in the lagrangian.

The bosonic lagrangian of the $D=10, \mathcal{N}=1$ abelian Yang-Mills supergravity which is coupled to $N U(1)$ gauge multiplets can be given as [4, 5, 6]

$$
\mathcal{L}_{10}=R * 1-\frac{1}{2} * d \phi_{1} \wedge d \phi_{1}-\frac{1}{2} e^{\phi_{1}} * F_{(3)} \wedge F_{(3)}-\frac{1}{2} e^{\frac{1}{2} \phi_{1}} \sum_{I=1}^{N} * G_{(2)}^{I} \wedge G_{(2)}^{I}
$$

where $G_{(2)}^{I}=d B_{(1)}^{I}$ are the $N U(1)$ gauge field strengths. In (2.1) $\phi_{1}$ is a scalar field and we have a two-form field $A_{(2)}$ whose field strength is defined 
as

$$
F_{(3)}=d A_{(2)}+\frac{1}{2} B_{(1)}^{I} \wedge d B_{(1)}^{I} .
$$

When the bosonic sector of the ten dimensional simple $\mathcal{N}=1$ supergravity which is coupled to $N$ abelian gauge multiplets is compactified on the Euclidean torus $T^{10-D}$ in $D$-dimensions one obtains the reduced lagrangian [6]

$$
\begin{aligned}
\mathcal{L}_{D}= & R * 1-\frac{1}{2} * d \phi \wedge d \phi+\frac{1}{4} \operatorname{tr}\left(* d \mathcal{M}^{-1} \wedge d \mathcal{M}\right) \\
& -\frac{1}{2} e^{-\sqrt{8 /(D-2)} \phi} * F_{(3)} \wedge F_{(3)}-\frac{1}{2} e^{-\sqrt{2 /(D-2)} \phi} * H_{(2)}^{T} \wedge \mathcal{M} H_{(2)}
\end{aligned}
$$

with

$$
H_{(2)}=d C_{(1)},
$$

where $C_{(1)}$ is a column vector of one-form fields whose dimension is (20$2 D+N)$. The field strength $F_{(3)}$ in $(2.3)$ is defined as

$$
F_{(3)}=d A_{(2)}+\frac{1}{2} C_{(1)}^{T} \Omega d C_{(1)}
$$

The definitions of the potentials used in writing (2.3) in terms of the original Kaluza-Klein potentials which emerge from the reduction ansatz used in (2.1) can be found in [6]. Apart form the single dilaton $\phi$ which is decoupled from the rest the scalars in (2.3) parametrize the coset

$$
\frac{O(10-D+N, 10-D)}{O(10-D+N) \times O(10-D)} .
$$


The $(20-2 D+N) \times(20-2 D+N)$ matrix $\Omega$ in (2.5) is

$$
\Omega=\left(\begin{array}{ccc}
0 & 0 & -\mathbf{1}_{(10-D)} \\
0 & \mathbf{1}_{(N)} & 0 \\
-\mathbf{1}_{(10-D)} & 0 & 0
\end{array}\right)
$$

where $\mathbf{1}_{(n)}$ is the $n \times n$ unit matrix. $\Omega$ is the invariant metric of $O(10-D+$ $N, 10-D)$. The scalar sector lagrangian in $(2.3)$ is based on the internal metric

$$
\mathcal{M}=\nu^{T} \nu
$$

where $\nu$ is the $O(10-D+N, 10-D) / O(10-D+N) \times O(10-D)$ coset representative. One can use the solvable Lie algebra parametrization [7, 8]

$$
\nu=e^{\frac{1}{2} \phi^{i} H_{i}} e^{\chi^{m} E_{m}}
$$

to parametrize the coset representative $\nu$. This parametrization is a result of the Iwasawa decomposition

$$
o(10-D+N, 10-D)=k_{0} \oplus s_{0}
$$

where $k_{0}$ is the Lie algebra of $O(10-D+N) \times O(10-D)$ which is a maximal compact subgroup of $O(10-D+N, 10-D)$ and $s_{0}$ is a solvable Lie subalgebra of $o(10-D+N, 10-D)$ [8, 9]. The set $\left\{H_{i}\right\}$ which is composed of a certain number of Cartan generators and the set $\left\{E_{m}\right\}$ which is composed of a certain number of positive root generators are the generators of $s_{0}[9]$. The $(10-D) \times(10-D+N)$ coset scalars are divided into the dilatons $\left\{\phi^{i}\right\}$ and the axions $\left\{\chi^{m}\right\}$.

As we have mentioned in the introduction in this work our aim is to derive a set of consistency conditions which reveal an implicit decoupling in the scalar-matter structure of (2.3). We should state that for observing the above mentioned consistency conditions we do not have to inspect the entire 
set of field equations of (2.3). It will be sufficient to take a look at only the field equation of the decoupled dilaton $\phi$ to obtain the necessary consistency conditions. Now if we vary the lagrangian (2.3) with respect to the scalar field $\phi$ we obtain the corresponding field equation as

$$
\begin{aligned}
(-1)^{D} d(* d \phi)= & \frac{1}{2} \sqrt{8 /(D-2)} e^{-\sqrt{\frac{8}{(D-2)} \phi} * F_{(3)} \wedge F_{(3)}} \\
& +\frac{1}{2} \sqrt{2 /(D-2)} e^{-\sqrt{\frac{2}{(D-2)}} \phi} \mathcal{M}_{i j} * H_{(2)}^{i} \wedge H_{(2)}^{j} .
\end{aligned}
$$

This equation can be written as

$$
-\sqrt{2 /(D-2)} \mathcal{L}_{m a t}=(-1)^{D} d(* d \phi)-\frac{1}{2} \sqrt{8 /(D-2)} e^{-\sqrt{\frac{8}{(D-2)}} \phi} * F_{(3)} \wedge F_{(3)},
$$

where

$$
\mathcal{L}_{\text {mat }}=-\frac{1}{2} e^{-\sqrt{\frac{2}{(D-2)}} \phi} \mathcal{M}_{i j} * H_{(2)}^{i} \wedge H_{(2)}^{j},
$$

is the term which couples the coset scalars to the matter gauge fields in (2.3). Now we should observe that since the right hand side of (2.12) does not depend on the dilatons and the axions if we take the partial derivative of both sides of (2.12) with respect to the dilatons $\left\{\phi^{i}\right\}$ and the axions $\left\{\chi^{m}\right\}$ we obtain

$$
\frac{\partial \mathcal{L}_{m a t}}{\partial \phi^{i}}=\frac{\partial \mathcal{L}_{m a t}}{\partial \chi^{m}}=0
$$

Since in deriving (2.14) we have started from the field equation (2.11) these conditions are consistency conditions which are satisfied by the solution space of (2.3). Before discussing their implications in the scalar sector we will work on them further more and derive the explicit form of the conditions (2.14). For any matrix function $\omega(x)$ we have [10, 11]

$$
\frac{\partial e^{\omega}}{\partial x}=e^{\omega}\left(\omega^{\prime}-\frac{1}{2 !}\left[\omega, \omega^{\prime}\right]+\frac{1}{3 !}\left[\omega,\left[\omega, \omega^{\prime}\right]\right]-\cdots\right),
$$


with

$$
\omega^{\prime} \equiv \frac{\partial \omega}{\partial x}
$$

When we choose a $(20-2 D+N)$-dimensional representation for $o(10-D+$ $N, 10-D)$ and express the generators $\left\{H_{i}\right\}$ and $\left\{E_{m}\right\}$ as matrices bearing in mind that the generators $\left\{H_{i}\right\}$ are Cartan generators [9] after some algebra one can show that

$$
\frac{\partial \nu}{\partial \phi^{i}}=\frac{1}{2} H_{i} \nu \quad \text { and } \quad \frac{\partial \nu^{T}}{\partial \phi^{i}}=\frac{1}{2} \nu^{T} H_{i}^{T}
$$

Therefore we find

$$
\frac{\partial \mathcal{M}}{\partial \phi^{i}}=\frac{1}{2}\left(\mathcal{A}+\mathcal{A}^{T}\right)
$$

where we define

$$
\mathcal{A}=\nu^{T} H_{i} \nu
$$

Now from (2.15) we also define

$$
\begin{aligned}
O\left(E_{m}\right) \equiv \frac{\partial\left(e^{\chi^{n} E_{n}}\right)}{\partial \chi^{m}}= & e^{\chi^{n} E_{n}}\left(E_{m}-\frac{1}{2 !}\left[\chi^{n} E_{n}, E_{m}\right]\right. \\
& \left.+\frac{1}{3 !}\left[\chi^{n} E_{n},\left[\chi^{t} E_{t}, E_{m}\right]\right]-\cdots\right),
\end{aligned}
$$

where we have used

$$
\frac{\partial\left(\chi^{n} E_{n}\right)}{\partial \chi^{m}}=E_{m}
$$

After some algebra one can prove that

$$
O^{T}\left(E_{m}\right)=O\left(E_{m}^{T}\right)
$$

By using the definition (2.20) and also the identity (2.22) similarly for the axions we find that

$$
\frac{\partial \mathcal{M}}{\partial \chi^{m}}=\mathcal{B}+\mathcal{B}^{T}
$$


where we introduce

$$
\mathcal{B}=\nu^{T} e^{\frac{1}{2} \phi^{i} H_{i}} O\left(E_{m}\right) .
$$

We should state that since $\left\{E_{m}\right\}$ are the generators of a nilpotent Lie subalgebra of $o(10-D+N, 10-D)[9]$ one can prove that the series in (2.20) should terminate after a finite number of terms [12, 13]. Thus the calculation of (2.24) is a straightforward task after choosing the representation. Now from (2.14) we have

$$
\begin{aligned}
& \frac{\partial \mathcal{L}_{m a t}}{\partial \phi^{i}}=-\frac{1}{2} e^{-\sqrt{\frac{2}{(D-2)}} \phi} \frac{\partial \mathcal{M}_{k l}}{\partial \phi^{i}} * H_{(2)}^{k} \wedge H_{(2)}^{l}=0, \\
& \frac{\partial \mathcal{L}_{m a t}}{\partial \chi^{m}}=-\frac{1}{2} e^{-\sqrt{\frac{2}{(D-2)}} \phi} \frac{\partial \mathcal{M}_{i j}}{\partial \chi^{m}} * H_{(2)}^{i} \wedge H_{(2)}^{j}=0 .
\end{aligned}
$$

By using (2.18) and (2.23) also by further simplifying we can finally write the consistency conditions in (2.25) as

$$
\begin{aligned}
& \mathcal{A}_{i j} * H_{(2)}^{i} \wedge H_{(2)}^{j}=0, \\
& \mathcal{B}_{i j} * H_{(2)}^{i} \wedge H_{(2)}^{j}=0 .
\end{aligned}
$$

Before concluding we should discuss how these conditions bring out an implicit decoupling between the coset scalars namely the dilatons and the axions and the $U(1)$ gauge fields. The scalar sector which governs the $(10-D) \times(10-D+N)$ coset scalars $\left\{\phi^{i}\right\}$ and $\left\{\chi^{m}\right\}$ in (2.3) can be given as

$$
\mathcal{L}\left(\phi^{i}, \chi^{m}\right)=\frac{1}{4} \operatorname{tr}\left(* d \mathcal{M}^{-1} \wedge d \mathcal{M}\right)+\mathcal{L}_{\text {mat }} .
$$

Now if we vary this lagrangian with respect to the dilatons $\left\{\phi^{i}\right\}$ and the axions $\left\{\chi^{m}\right\}$ to find the corresponding field equations we immediately see that since the matter lagrangian in (2.27) does not depend on the field strengths of the dilatons and the axions and moreover due to the conditions (2.14) 
which are satisfied by the elements of the solution space the dilaton and the axion field equations are equivalent to the ones which would be obtained by directly varying the pure $G / K$ coset sigma model lagrangian

$$
\mathcal{L}_{\text {purescalar }}=\frac{1}{4} \operatorname{tr}\left(* d \mathcal{M}^{-1} \wedge d \mathcal{M}\right) .
$$

Thus we prove that the coset scalar solutions of the field equations of (2.3) are contained in the general solution space of the pure symmetric space sigma model lagrangian (2.28), 1 . This may be identified as an implicit decoupling structure of the coset scalars and the abelian gauge fields $C_{(1)}$. Therefore we conclude that although there is a coupling between the coset scalars and the abelian gauge fields at the lagrangian level in (2.3) the consistency conditions we have derived in (2.14) reflect an implicit decoupling between the coset scalars and the abelian gauge fields in the coset scalar field equations. However we should state that the coset scalars and the rest of the fields are still coupled to each other in the other field equations. Thus as a result we observe that the dilaton and the axion solutions of the field equations of the $D$-dimensional lagrangian (2.3) form up a subset of the pure scalar coset sigma model solution space.

\section{Conclusion}

By inspecting the decoupled dilaton field equation of the bosonic lagrangian of the $D$-dimensional fully Higgsed low energy effective heterotic string [6] we have derived two sets of consistency conditions which are satisfied by the elements of the bosonic solution space. Assuming the solvable Lie algebra parametrization for the scalar coset manifold and a matrix representation for the global symmetry algebra we have obtained the explicit forms of these conditions in terms of the dilatons and the axions. Furthermore we have

\footnotetext{
${ }^{1}$ To see how the field equations of the pure symmetric space sigma model can be derived one may refer to [9, 14].
} 
discussed that these conditions bring out a hidden decoupling between the coset scalars and the gauge fields of the theory from the scalar solution space point of view despite the existence of a coupling term in the lagrangian. Therefore we have revealed an implicit constraint which shapes the bosonic solution space of the fully Higgsed effective heterotic string.

The consistency conditions we have obtained can be effectively used to generate ansatz for solving the bosonic field equations of the theory. For this reason we have also derived the explicit form of these conditions in the solvable Lie algebra gauge of the scalar coset manifold. Besides we have shown that the coset scalar solutions of the $D$-dimensional fully Higgsed low energy effective heterotic string are embedded in the solution space of the pure symmetric space $G / K$ sigma model. Thus the general solutions of the pure coset scalar sector can also be used as ansatz in solving the bosonic field equations of the $D$-dimensional effective heterotic string.

The main achievement of this work is to reveal the fact that the single dilaton which behaves distinctively in the $D$-dimensional effective heterotic string lagrangian generates a scalar-matter decoupling structure for the content of the scalar solution space and its existence contributes an important set of consistency conditions on the entire bosonic solution space. Such an implicit decoupling of the coset scalars from the rest of the bosonic field content also carries implications why the global symmetry of the pure coset scalar sector can be extended to be the global symmetry of the entire bosonic sector. Therefore this work may help us to understand the global symmetries of the supergravities thus the duality scheme of the string theory better.

Although we have assumed the solvable Lie algebra gauge to parametrize the scalar coset manifold of the $D$-dimensional theory the consistency conditions we have derived are valid for any parametrization, scalar field definition and the formulation of the coset scalar sector lagrangian. Therefore these conditions can be studied further more in different formulations of the scalar sector. We should state that the decoupling studied in this work is a pseudo 
one instead since when one considers the rest of the bosonic field equations of the theory one sees that the coset scalars take part in the equations. Thus the solution configuration of the coset scalars do depend on the other fields since they couple to the rest of the field content in the other field equations. However the field equations of the coset scalars can be decoupled from the rest of the fields denoting that the coset scalar solution space is contained in the pure scalar sector non-linear sigma model solution space. With the perspective of understanding the global symmetry structure of the supergravities we have focussed on the role of the scalar coset sigma model in the bosonic theory. For this reason we have inspected its bosonic couplings and we have omitted the fermionic sector. One may do a similar search to derive consistency conditions which would reveal the relations between the pure scalar sector and its coupling extensions in the entire theory.

\section{References}

[1] E. Kiritsis, "Introduction to superstring theory", hep-th/9709062.

[2] C. M. Hull and P. K. Townsend, "Unity of superstring dualities", Nucl. Phys. B438 (1995) 109, hep-th/9410167.

[3] E. Witten, "String theory dynamics in various dimensions", Nucl. Phys. B443 (1995) 85, hep-th/9503124.

[4] E. Bergshoeff, M. de Roo, B. de Wit and P. van Nieuwenhuizen, "Tendimensional Maxwell-Einstein supergravity, its currents, and the issue of its auxiliary fields", Nucl. Phys. B195 (1982) 97.

[5] G. F. Chapline and N. S. Manton, "Unification of Yang-Mills theory and supergravity in ten-dimensions", Phys. Lett. B120 (1983) 105. 
[6] H. Lü, C. N. Pope and K. S. Stelle, "M-theory/heterotic duality: A Kaluza-Klein perspective", Nucl. Phys. B548 (1999) 87, hep-th/9810159.

[7] L. Andrianopoli, R. D'Auria, S. Ferrara, P. Fre, M. Trigiante, " $R-R$ scalars, U-duality and solvable Lie algebras", Nucl. Phys. B496 (1997) 617, hep-th/9611014.

[8] S. Helgason, "Differential Geometry, Lie Groups and Symmetric Spaces", (Graduate Studies in Mathematics 34, American Mathematical Society Providence R.I. 2001).

[9] N. T. Yllmaz, "The non-split scalar coset in supergravity theories", Nucl. Phys. B675 (2003) 122, hep-th/0407006.

[10] D. H. Sattinger, O. L. Weaver, "Lie Groups and Algebras with Applications to Physics, Geometry and Mechanics", (SpringerVerlag New York, Inc. 1986).

[11] B. C. Hall, "Lie Groups, Lie Algebras, and Representations: An Elementary Introduction", (Graduate Texts in Mathematics, 222, Springer-Verlag New York, Inc. 2003).

[12] R. Carter, G. Segal, I. MacDonald, "Lectures on Lie Groups and Lie Algebras", (London Mathematical Society Student Texts, 32, Cambridge University Press 1995).

[13] A. L. Onishchik (Ed.), V. V. Gorbatsevich, E. B. Vinberg, "Lie Groups and Lie Algebras I", (Springer-Verlag New York, Inc. 1993).

[14] A. Keurentjes, "The group theory of oxidation II : Cosets of non-split groups", Nucl. Phys. B658 (2003) 348, hep-th/0212024. 